\title{
Effectiveness of High-Fidelity Simulation in Training Emergency Medicine Physicians in Point of Care Ultrasonography in Pakistan: A Quasi- Experimental Study
}

\author{
Kiran Azizi ${ }^{1}$, Madiha Ismail ${ }^{1}$, Umaira Aftab ${ }^{1}$, Badar Afzal ${ }^{1}$, Asad Mian ${ }^{2}$ \\ 1. Emergency Medicine, Aga Khan University, Karachi, PAK 2. Pediatric Emergency Medicine, Aga Khan University, \\ Karachi, PAK
}

Corresponding author: Kiran Azizi, azizi333@live.com

\begin{abstract}
\section{Background}

Point-of-care ultrasound (PoCUS) is frequently utilized in emergency medicine (EM), with an extendedfocused assessment with sonography in trauma (e-FAST) being the most widely used PoCUS modality. This modality is not only time- and cost-efficient, but it is highly accurate in the diagnosis and management of surgical patients in the emergency department, as well as being highly predictive of patient outcomes. Targeted training is essential to ensure a learner's confidence in image acquisition, interpretation, and translation of knowledge to making clinical decisions. High-fidelity simulation offers a uniquely safe and "mistake-forgiving" environment to teach and train medical professionals. The present study evaluated the effectiveness of a high-fidelity simulator to train EM physicians in e-FAST at a tertiary care teaching hospital in a lower-middle-income country.
\end{abstract}

\section{Methods}

This quasi-experimental study was performed at a state-of-the-art simulation center of a multidisciplinary university hospital in Karachi, Pakistan. Subjects were included if they were EM physicians who volunteered to participate and were available for the entire training and testing period. The educational intervention included lectures and hands-on practice on a high-fidelity simulator (SonoSim, Santa Monica, CA).

Received 05/29/2020

Review began 06/09/2020 Review ended 06/10/2020 Published 06/17/2020

() Copyright 2020 Azizi et al. This is an open access article distributed under the terms of the Creative Commons Attribution License CC-BY 4.0., which permits unrestricted use, distribution, and reproduction in any medium, provided the original author and source are credited.
Knowledge and image interpretation on e-FAST were evaluated using a questionnaire, administered before and after the training course. Each participant's ability to acquire and interpret satisfactory images was assessed by experienced EM physicians and recorded. Participants were also administered a needs assessment survey and a course evaluation. Data were analyzed using IBM SPSS Statistics for Windows, Version 20.0 (Armonk, NY: IBM Corp.). All the tests were two-sided, and p-values $\leqslant 0.05$ were considered significant. Baseline characteristics and outcome variables were recorded and compared by Wilcoxon signedrank tests.

\section{Results}

A total of 31 EM physicians, 12 (38.7\%) men and 19 (61.3\%) women, were enrolled in the study, with 24 (77.3\%) having one to three years of EM experience. Mean and percentage group performance improved from 6 and $40 \%$ before the intervention to 14.5 and 96.6\% after the intervention ( $\mathrm{Z}=4.867, \mathrm{p} \leqslant 0.05)$. Most improvement in image acquisition on high-fidelity simulation was observed in the upper right quadrant of the suprapubic window $(29 / 31 ; 93.5 \%)$, followed by the upper left quadrant $(27 / 31 ; 87 \%)$ and the subxiphoid window $(21 / 31 ; 67 \%)$. All 31 participants reported improvements in comfort and confidence level with PoCUS after attending the workshop.

\section{Conclusions}

EM physicians who attended a brief workshop incorporating simulation demonstrated improvements in knowledge and image acquisition skills in all domains tested. High-fidelity simulation training is an effective modality for training EM physicians in e-FAST.

Categories: Emergency Medicine, Miscellaneous, Trauma

Keywords: pocus, emergency medicine physician, high fidelity simulation, skill training, efast

\section{Introduction}

Point-of-care ultrasound (PoCUS) is defined as ultrasonography at the patient's bedside performed in real time by a care provider [1]. Focused assessment with sonography for trauma (FAST) is an integrated, goaldirected, bedside examination performed to detect fluid, which is likely to be hemorrhage in cases of trauma 
[2]. In addition to pericardial and peritoneal windows, extended FAST (e-FAST) also includes an examination of the chest. Studies from the developed world have shown that e-FAST is an effective and sensitive technique for the detection of blunt abdominal trauma and that it is equally accurate when administered by radiologists and non-radiologists [3].

Similar to other aspects of physical examinations, PoCUS is dependent on the operator [4]. Confidence and competence in both image acquisition and interpretation are essential, as is knowledge of how to incorporate findings into clinical decisions [5]. Because optimal training of physicians is necessary, many institutions worldwide include PoCUS in their undergraduate and postgraduate training and curricula [6]. Little is known, however, about the ability of physicians to acquire e-FAST skills using high-fidelity simulators to diagnose life-threatening conditions in trauma patients [7,8]. Moreover, the feasibility and effectiveness of this program in a lower-middle-income country (LMIC) like Pakistan have not been determined [2,3]. The present study assessed the knowledge and skills of emergency medicine (EM) physicians after a brief training workshop on e-FAST, including high-fidelity simulators, in a tertiary care teaching hospital in Karachi, Pakistan.

\section{Materials And Methods}

This quasi-experimental study enrolled EM physicians, including postgraduate medical trainees (residents) and non-trainees (medical officers and senior medical officers), working in an urban tertiary care university hospital. The study was approved by the ethical review committee of Aga Khan University and was conducted in a simulation center located in a multidisciplinary university hospital in Karachi, Pakistan. All EM physicians at the hospital were invited to participate, with all those agreeing and enrolled providing written informed consent.

Participants were eligible if they were EM physicians who volunteered to participate, and were available for the entire training and testing period. Each physician participated in a half-day (3.5-hour) workshop on PoCUS-eFAST, which included didactic lectures on knobology, knowledge of image acquisition, and knowledge of image interpretation, followed by a hands-on practice session and then assessment on a highfidelity simulator (SonoSim, Santa Monica, CA) and by a human volunteer (Figure 1).

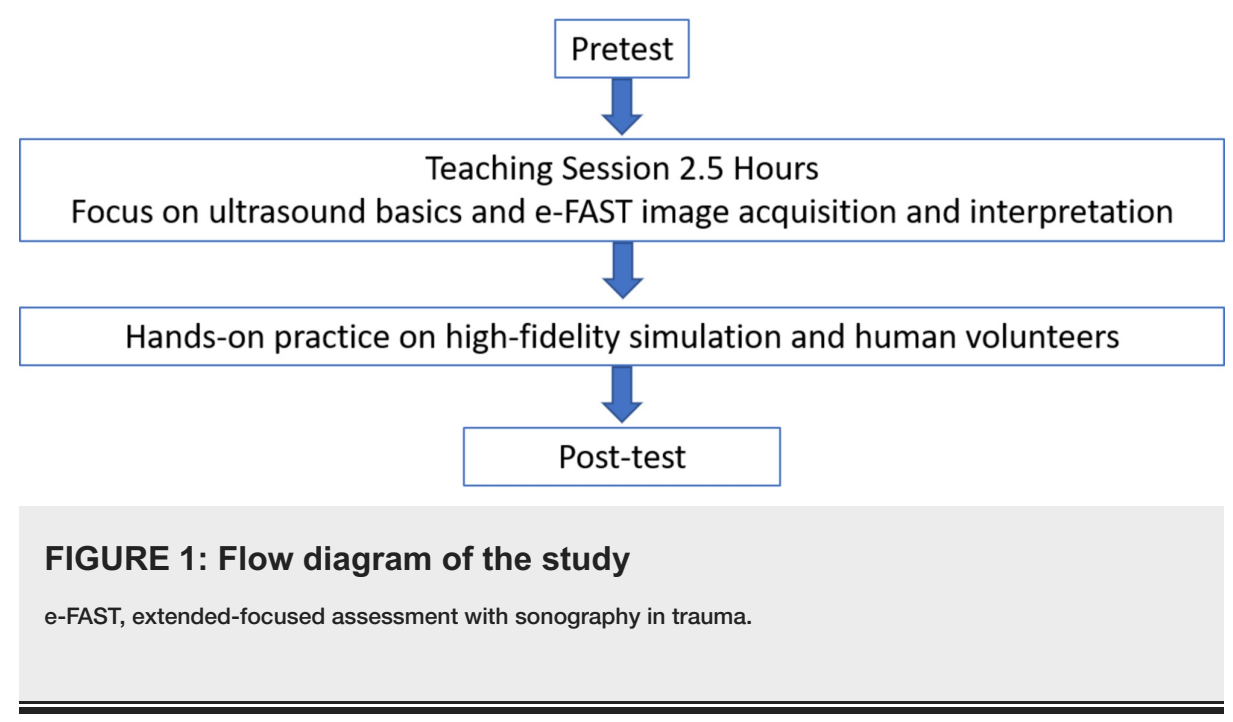

Knowledge regarding image acquisition and interpretation skills was evaluated by a questionnaire and by experienced EM physicians. The preintervention questionnaire included questions about demographic characteristics, need assessments, cognitive skills and interpretation of e-FAST images, and barriers to PoCUS practice. After the training session, the participants were administered the same questionnaire, which included questions about subjects' knowledge and feedback on the workshop. To assess changes in knowledge, each trainee's results before and after the training session were compared. Data were compiled anonymously by a blinded reviewer and analyzed statistically using IBM SPSS Statistics for Windows, Version 20.0 (Armonk, NY: IBM Corp.).

All statistical analyses were two-sided, with p-values $\leqslant 0.05$ considered statistically significant. Normally distributed continuous variables were reported as mean \pm standard deviation, and skewed variables were reported as median and range. Results obtained before and after the training session were compared by the Wilcoxon signed-rank test. Generalized estimation equation analysis was used to determine factors influencing the variability in scores before and after the intervention.

\section{Results}

The study enrolled 31 qualified medical practitioners, including 12 (38.7\%) men and 19 (61.3\%) women, with 


\section{Cureus}

$24(77.4 \%)$ having one to three years of work experience. Eight (27.6\%) were residents, 11 (38\%) were medical officers, and 10 (34.5\%) were senior medical officers. Only eight (26\%) participants had previously received formal ultrasound training. Although 29 (93.6\%) reported that an ultrasound machine was easily available in the emergency department, only three (9.86\%) were using it routinely for FAST examinations. A total of 24 participants (77.4\%) identified a lack of training, and 18 (58.1\%) identified a lack of knowledge as the most important barriers to PoCUS utilization. Following training, all 31 (100\%) self-reported improvement in levels of comfort and confidence with PoCUS, with 20 (64\%) stating that ultrasound teaching sessions should take place on monthly (Table 1).

\begin{tabular}{|l|l|}
\hline Variables & $\mathrm{n}(\%)$ \\
\hline Gender & $12(38.7)$ \\
\hline Male & $19(61.3)$ \\
Pemale & 8(27.7) \\
Resident & $11(37.9)$ \\
Medical officer & $10(34.5)$ \\
Senior medical officer & \\
Duration practicing in the emergency department & $24(77.4)$ \\
$1-3$ years & $3(9.7)$ \\
$4-5$ years & $4(12.9)$ \\
\hline$\geq 5$ years & \\
\hline
\end{tabular}

TABLE 1: Baseline characteristics of the study participants ( $N=31)$

A comparison of scores before and after training showed significant improvements (Table 2), with mean group performance improving from 6 to 14.5 , and percentage improving from $40 \%$ to $96.6 \%$ ( $Z=4.867, p<0.05$ by Wilcoxon signed-rank tests; Table 3). 


\section{Cureus}

\begin{tabular}{|c|c|c|c|c|}
\hline Questions & $\begin{array}{l}\text { Before training } \\
\text { session }\end{array}$ & $\begin{array}{l}\text { After training } \\
\text { session }\end{array}$ & $\begin{array}{l}\text { P- } \\
\text { value }\end{array}$ & $\begin{array}{l}\text { Confidence } \\
\text { interval }\end{array}$ \\
\hline What does FAST stand for? & $20(64.5)$ & $29(93.5)$ & 0.002 & $-6.01,-1.36$ \\
\hline $\begin{array}{l}\text { Name all four windows used for evaluation of free fluid in FAST } \\
\text { exam }\end{array}$ & $18(58.1)$ & $31(100.0)$ & $<0.001$ & $4.81,8.71$ \\
\hline What does e-FAST stand for? & $12(38.7)$ & $29(93.5)$ & $<0.001$ & $4.58,8.25$ \\
\hline Which windows are added in e-FAST exam? & $9(29.0)$ & $30(96.8)$ & $<0.001$ & $5.31,8.86$ \\
\hline Ideal probe for FAST exam? & $19(61.3)$ & $31(100.0)$ & 0.010 & $0.75,5.67$ \\
\hline Amount of blood detected by FAST & $24(77.4)$ & $31(100.0)$ & $<0.001$ & $3.47,8.73$ \\
\hline Most dependent point of peritoneal cavity & $18(58.1)$ & $28(90.3)$ & $<0.001$ & $1.98,5.80$ \\
\hline Pneumothorax sign on sonography & $7(22.6)$ & $24(77.4)$ & 0.112 & $-0.41,3.98$ \\
\hline Identification of suprapubic window & $12(38.7)$ & $30(96.8)$ & $<0.001$ & $4.83,8.18$ \\
\hline Free fluid (suprapubic window) & $18(58.1)$ & $30(96.8)$ & $<0.001$ & $4.33,8.01$ \\
\hline Identification of Morrison's pouch & $14(45.2)$ & $30(96.8)$ & $<0.001$ & $5.49,8.52$ \\
\hline Free fluid (Morrison's pouch) & $15(48.4)$ & $29(93.5)$ & $<0.001$ & $4.01,7.03$ \\
\hline
\end{tabular}

TABLE 2: Comparison of correct responses by participants to questions before and after the training session $(\mathrm{N}=31)$

e-FAST, extended-focused assessment with sonography in trauma; FAST, focussed assessment with sonography in trauma.

\begin{tabular}{|c|c|c|c|c|c|c|}
\hline Variables & Mean & Median & Standard deviation & Difference & Z-Score & P-value \\
\hline Before training & 6.58 & 6 & 4.02 & \multirow{2}{*}{251} & \multirow{2}{*}{$4.867^{\dagger}$} & \multirow{2}{*}{$<0.001^{*}$} \\
\hline After training & 14.67 & 15 & 0.65 & & & \\
\hline
\end{tabular}

TABLE 3: Comparison of correct responses by participants to questions before and after the training session $(\mathrm{N}=31)$

* Wilcoxon signed-rank test.

† Based on positive ranks.

Generalized estimation equation analysis of factors influencing the variability in scores before and after intervention showed that high scores were more likely to be achieved by participants in their mid-level career stage, with work experience of four to five years (Table 4). Also, those identifying lack of training as the most significant barrier to PoCUS utilizations scored higher on postintervention tests (Table 4). 


\section{Cureus}

\begin{tabular}{|c|c|c|c|}
\hline Variables & $\begin{array}{l}\beta \text {-coefficient (S.E } \\
\text { (ß)) }\end{array}$ & $\begin{array}{l}\text { P- } \\
\text { value }\end{array}$ & $\begin{array}{l}\text { Confidence } \\
\text { interval }\end{array}$ \\
\hline \multicolumn{4}{|l|}{ Duration practicing in the emergency department } \\
\hline $1-3$ years & - & - & - \\
\hline 4-5 years & $2.66(0.88)$ & $0.003^{*}$ & $0.92,4.39$ \\
\hline$\geq 5$ years & $-.41(0.94)$ & 0.134 & $-3.27,0.43$ \\
\hline \multicolumn{4}{|l|}{ Physician level in the emergency department } \\
\hline Resident & - & - & - \\
\hline Medical officer & $-2.20(0.70)$ & $0.002^{\star}$ & $-3.57,-0.82$ \\
\hline Senior medical officer & $-0.66(0.77)$ & 0.389 & $-2.18,0.85$ \\
\hline $\begin{array}{l}\text { In my opinion, "Lack of training" is the biggest barrier to utilizing PoCUS in the emergency } \\
\text { department }\end{array}$ & $2.08(0.68)$ & $0.002^{*}$ & $0.74,3.42$ \\
\hline
\end{tabular}

TABLE 4: Statistical comparison of test scores before and after the training session using a generalized estimating equation test model

* Significant P-values.

PoCUS, point-of-care ultrasound. S.E. $\beta$, standard error $\beta$.

Physicians were inquired about the needs assessment of PoCUS in emergency department (Table 5).

\begin{tabular}{|c|c|}
\hline PoCUS utilization in the ED & n (\%) \\
\hline An ultrasound machine is easily available in my department & $29(93.5)$ \\
\hline \multicolumn{2}{|l|}{ I frequently use PoCUS } \\
\hline Strongly disagree & $3(9.78)$ \\
\hline Disagree & $4(12.9)$ \\
\hline Neutral & $22(71.0)$ \\
\hline Agree & $2(6.5)$ \\
\hline \multicolumn{2}{|l|}{ Strongly agree } \\
\hline \multicolumn{2}{|l|}{ I have received formal training on PoCUS in the past } \\
\hline Yes & $8(25.8)$ \\
\hline No & $23(74.2)$ \\
\hline \multicolumn{2}{|l|}{ I frequently use ultrasound for FAST exam } \\
\hline Strongly disagree & $6(19.4)$ \\
\hline Disagree & $8(25.8)$ \\
\hline Neutral & $13(41.9)$ \\
\hline Agree & $2(6.5)$ \\
\hline Strongly agree & 1 (3.2) \\
\hline Missing & $1(3.2)$ \\
\hline \multicolumn{2}{|l|}{ I frequently use ultrasound to look for pneumothorax } \\
\hline Strongly disagree & $13(41.9)$ \\
\hline
\end{tabular}


Cureus

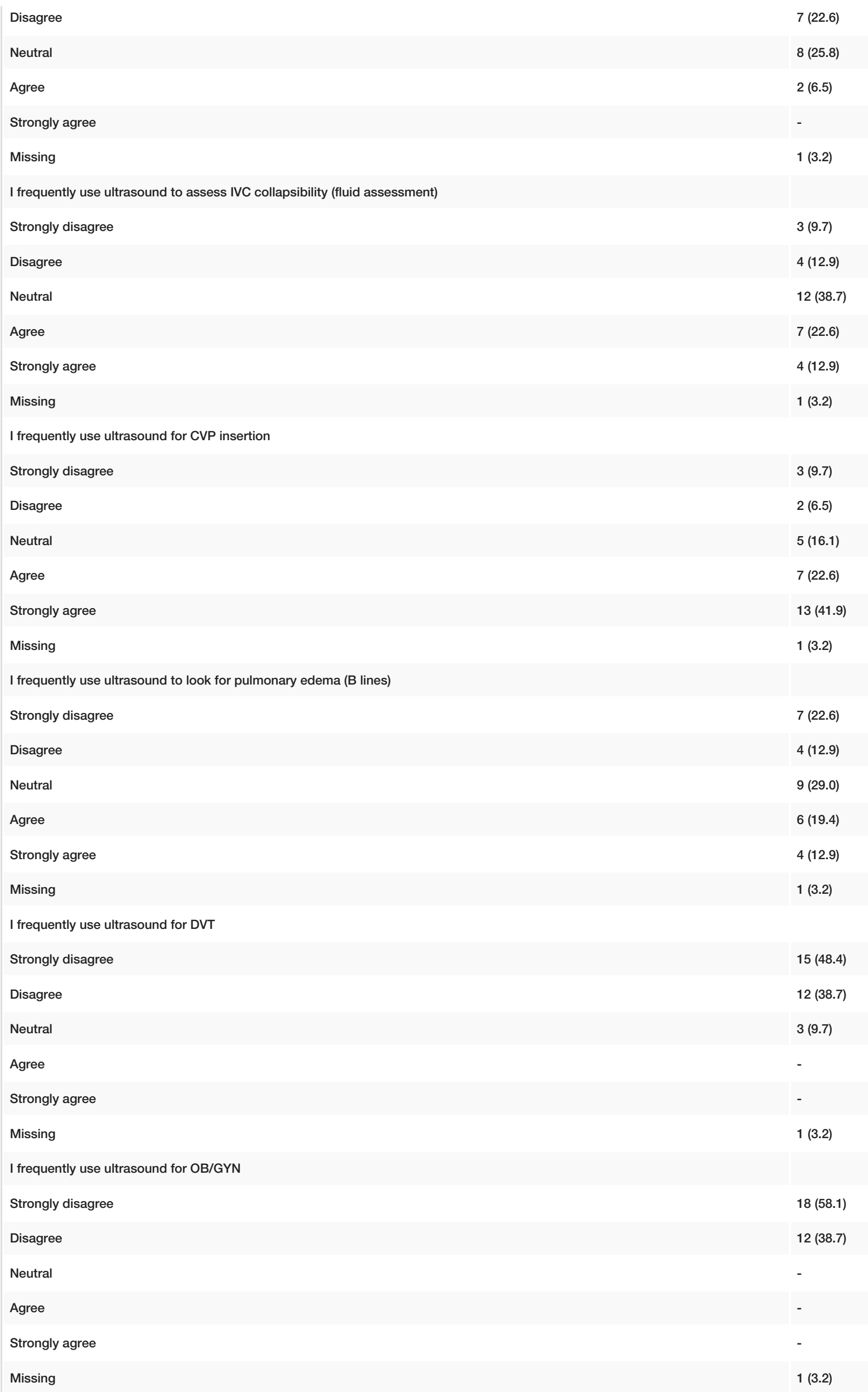

2020 Azizi et al. Cureus 12(6): e8659. DOI 10.7759/cureus.8659

6 of 10 


\section{Cureus}

In my opinion, "shortage of time" is the biggest barrier to utilizing PoCUS in the ED

Agree

In my opinion, "lack of training" is the biggest barrier to utilizing PoCUS in the ED

Agree

In my opinion, "shortage of staff" is the biggest barrier to utilizing PoCUS in the ED

Agree

Disagree

In my opinion, "limited knowledge by physicians" is the biggest barrier to utilizing PoCUS in the ED

Agree

Disagree

This workshop has increased my level of comfort and confidence in PoCUS

Strongly disagree

Disagree

Neutral

Agree

Strongly agree

How frequently should ultrasound sessions be held?

Monthly

$20(64.5)$

Quarterly

Half yearly

Yearly

\section{TABLE 5: Needs assessment of PoCUS in the ED}

PoCUS, point-of-care ultrasound; FAST, focused assessment with sonography in trauma; IVC, inferior vena cava; CVP, central venous pressure; DVT, deep vein thrombosis; OB/GYN, obstetrics/gynecology; ED, emergency department.

Participants were tested on four trauma scenarios using a high-fidelity simulator (SonoSim). Images of the right upper quadrant, left upper quadrant, and subxiphoid and suprapubic windows were accurately acquired by 29 (93.5\%), 27 (87\%), 27 (87\%), and 29 (93.5\%) participants, respectively, whereas images of these windows were correctly interpreted by 24 (77.4\%), 27 (87\%), 19 (61.3\%), and 20 (64.5\%) subjects, respectively (Figure 2). 


\section{Cureus}

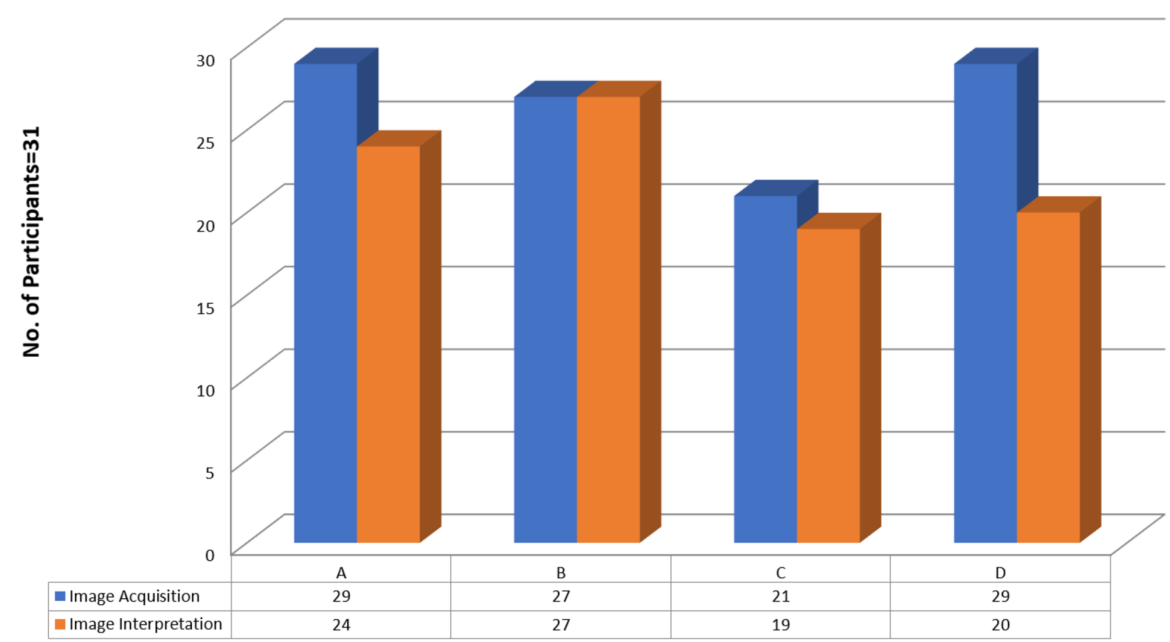

FIGURE 2: Correct image acquisition and interpretation, as determined by high-fidelity simulation

When evaluated by an experienced EM physician, images of the right upper quadrant, left upper quadrant, subxiphoid, suprapubic and pleural windows were accurately acquired by 22 (70.9\%), 23 (74.2\%), 20 (64.5\%), 20 (64.5\%), and 22 (70.9\%) participants, respectively (Figure 3).

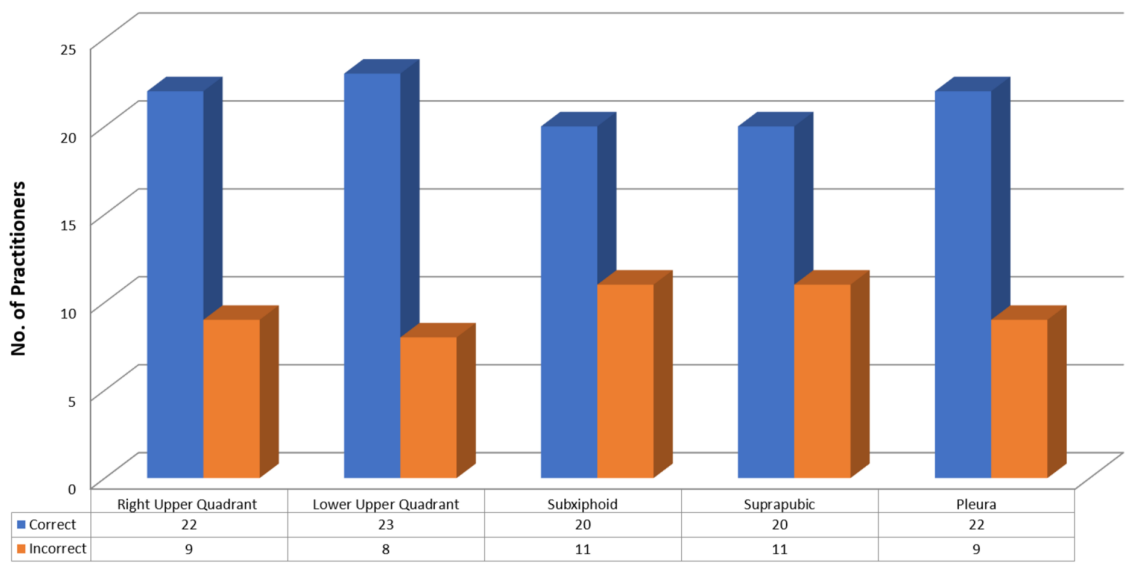

FIGURE 3: Correct image acquisition, as determined by an experienced emergency medicine physician

\section{Discussion}

This study showed that a high-fidelity simulation-based workshop improved the knowledge and skills of emergency physicians performing e-FAST. Learners displayed a significant improvement in written posttraining scores that assessed knowledge and image interpretation abilities. Although subjects participated in a half-day workshop focused on e-FAST, their post-training test scores were comparable to those of a threeday PoCUS workshop [9]. This is important for educators when planning skill-based simulation courses, as even a short course improved knowledge and interpretation of images by EM physicians. Secondary generalized estimation equation analysis showed that physicians with four to five years of experience did better on test scores than those with more than five years of experience, confirming earlier findings [9].

All 31 participants reported subjective improvements in comfort and confidence level regarding the use of eFAST at the bedside, comparable to previous results [10]. A positive correlation has been observed between confidence and knowledge [11]. Moreover, both can decay after initial training and can be regained after refresher courses [12]. 
Pre-training needs assessment found that, despite the availability of an ultrasound machine, it was mostly used for central venous line access and fluid assessment only, whereas other modalities, such as bedside echo and FAST, were mostly underutilized. This observation is not only consistent with other studies from LMICs but also highlighted the urgent need of integrating PoCUS into the EM residency curriculum in developing countries [8].

Similar to previous studies, the major barriers to PoCUS utilization identified by the participants in our were lack of training and limited knowledge [13,14]. The introduction of longitudinal teaching programs for both trainees and non-trainee physicians in EM may not only enhance their knowledge but result in better patient-centered outcomes $[11,15]$.

Additional studies are needed to ascertain the optimal duration of high-fidelity-based simulation courses on PoCUS. In most developed countries, physicians participate in two- to three-day, multiple level sign-out courses in ultrasound [11]. However, two-hour sessions may be beneficial, with flattening of operators' learning curves after 10 to 30 examinations [16-18]. Moreover, written tests of knowledge regarding the use of PoCUS do not correlate with procedural skills, suggesting that evaluation of both skillsets may be necessary to tailor training [19].

\section{Limitations}

This study had several limitations. First, it was performed at a single academic center with a small group of participants, with all participants being invited volunteers. Secondly, we did not evaluate hands-on skills before the training session; therefore, improvements in image acquisition could not be evaluated. Also, retention and application of knowledge in clinical settings were not evaluated (Kirkpatrick's level 3 evaluation), and the confidence level was assessed only after the training session [20]. Although we observed a positive outcome, we did not compare outcomes after our 3.5-hour session with those after other courses of different lengths. Thus, an optimal course length requires further evaluation. Additional studies are needed to determine long-term retention of knowledge and skills after training and to determine whether knowledge gained in a simulation setting is used in real-life clinical practice.

\section{Conclusions}

PoCUS is not being used to its full potential in emergency departments of LMICs. The major barriers are the lack of training and knowledge. High-fidelity simulation training is effective in training EM physicians in eFAST, with a half-day workshop that included simulations resulting in significant improvements in written test scores that assessed cognitive and image interpretation abilities. Further studies are needed to determine whether knowledge gained in simulation settings can be translated into clinical practice.

\section{Additional Information \\ Disclosures}

Human subjects: Consent was obtained by all participants in this study. Ethical Review Committee Aga Khan University Hospital issued approval 2019-0882-2832. After ethical review committee approval data collection was done. Animal subjects: All authors have confirmed that this study did not involve animal subjects or tissue. Conflicts of interest: In compliance with the ICMJE uniform disclosure form, all authors declare the following: Payment/services info: All authors have declared that no financial support was received from any organization for the submitted work. Financial relationships: All authors have declared that they have no financial relationships at present or within the previous three years with any organizations that might have an interest in the submitted work. Other relationships: All authors have declared that there are no other relationships or activities that could appear to have influenced the submitted work.

\section{Acknowledgements}

I would like to acknowledge Dr.Afsheen Ghani for facilitating us in completing this research project.

\section{References}

1. Moore CL, Copel JA : Point-of-care ultrasonography. N Engl J Med. 2011, 364:749-757. 10.1056/NEJMra0909487

2. Scalea TM, Rodriguez A, Chiu WC, et al.: Focused assessment with sonography for trauma (FAST): results from an international consensus conference. J Trauma. 1999, 46:466-472. 10.1097/00005373-19990300000022

3. Kirkpatrick AW, Sirois M, Laupland KB, et al.: Hand-held thoracic sonography for detecting post-traumatic pneumothoraces: the extended focused assessment with sonography for trauma (EFAST). J Trauma. 2004, 57:288-295. 10.1097/01.TA.0000133565.88871.E4

4. Lewiss RE, Hoffmann B, Beaulieu Y, Phelan MB: Point-of-care ultrasound education: the increasing role of simulation and multimedia resources. J Ultrasound Med. 2014, 33:27-32. 10.7863/ultra.33.1.27

5. Breitkreutz R, Uddin S, Steiger H, et al.: Focused echocardiography entry level: new concept of a 1-day training course. Minerva Anestesiol. 2009, 75:285-292. 
6. Orr KE, Hamilton SC, Clarke R, et al.: The integration of transabdominal ultrasound simulators into an ultrasound curriculum. Ultrasound. 2019, 27:20-30. 10.1177/1742271X18762251

7. Geria RN, Raio CC, Tayal V: Point-of-care ultrasound: not a stethoscope-a separate clinical entity . J Ultrasound Med. 2015, 34:172-173. 10.7863/ultra.34.1.172

8. Fagley RE, Haney MF, Beraud AS, et al.: Critical care basic ultrasound learning goals for American anesthesiology critical care trainees: recommendations from an expert group. Anesth Analg. 2015, 120:1041-1053. 10.1213/ANE.0000000000000652

9. Greenstein YY, Littauer R, Narasimhan M, Mayo PH, Koenig SJ: Effectiveness of a critical care ultrasonography course. Chest. 2017, 151:34-40. 10.1016/j.chest.2016.08.1465

10. Piposar JR, Easley M, Nunley JA, et al.: Musculoskeletal ultrasound education: orthopaedic resident ability following a multimedia tutorial. J Surg Orthop Adv. 2015, 24:64-68.

11. Safavi AH, Shi Q, Ding M, et al.: Structured, small-group hands-on teaching sessions improve pre-clerk knowledge and confidence in point-of-care ultrasound use and interpretation. Cureus. 2018, 10:e3484. 10.7759/cureus.3484

12. Schwid M, Harris O, Landry A, Eyre A, Henwood P, Kimberly H: Use of a refresher course increases confidence in point-of-care ultrasound skills in emergency medicine faculty. Cureus. 2019, 11:e5413. 10.7759/cureus. 5413

13. Ailon J, Mourad O, Nadjafi M, Cavalcanti R: Point-of-care ultrasound as a competency for general internists: a survey of internal medicine training programs in Canada. Can Med Educ J. 2016, 7:51-69.

14. Eisen LA, Leung S, Gallagher AE, Kvetan V: Barriers to ultrasound training in critical care medicine fellowships: a survey of program directors. Crit Care Med. 2010, 38:1978-1983. 10.1097/CCM.0b013e3181eeda53

15. Yamada T, Minami T, Soni NJ, et al.: Skills acquisition for novice learners after a point-of-care ultrasound course: does clinical rank matter?. BMC Med Educ. 2018, 18:202. 10.1186/s12909-018-1310-3

16. Davis VW, Wallace JM, Ahern MT, et al.: Mid-level providers demonstrate proficiency in FAST after directed training. Crit Ultrasound J. 2011, 3:111-113. 10.1007/s13089-011-0070-7

17. Gracias VH, Frankel HL, Gupta R, et al.: Defining the learning curve for the focused abdominal sonogram for trauma (FAST) examination: implications for credentialing. Am Surg. 2001, 67:364-368.

18. Choi YJ, Jung JY, Kwon H: Effectiveness of education in point-of-care ultrasound-assisted physical examinations in an emergency department: a before-and-after study. Medicine. 2017, 96:e7269. 10.1097/MD.0000000000007269

19. Kirkpatrick JD, Kirkpatrick WK: Kirkpatrick's Four Levels of Training Evaluation . Association for Talent Development, Alexandria, VA; 2016.

20. Stolz LA, Amini R, Situ-LaCasse E, et al.: Multimodular ultrasound orientation: residents' confidence and skill in performing point-of-care ultrasound. Cureus. 2018, 10:e3597. 10.7759/cureus.3597 